\title{
A Single Objective Flower Pollination Algorithm for Modeling the Horizontal Flexible Plate System
}

\author{
Siti Zakiah Maseri \\ Faculty of Mechanical Engineering \\ Universiti Teknologi MARA \\ Shah Alam, Malaysia \\ sitizakiahmaseri@gmail.com \\ Hanim Mohd Yatim \\ School of Mechanical Engineering \\ Universiti Teknologi Malaysia \\ Johor, Malaysia \\ hanim.my@utm.my
}

\author{
*Muhamad Sukri Hadi \\ Faculty of Mechanical Engineering \\ Universiti Teknologi MARA \\ Shah Alam, Malaysia \\ msukrihadi@uitm.edu.my \\ Mat Hussin Ab Talib \\ School of Mechanical Engineering \\ Universiti Teknologi Malaysia \\ Johor, Malaysia \\ mathussin@utm.my
}

\author{
Annisa Jamali \\ Faculty of Engineering \\ Universiti Malaysia Sarawak \\ Kota Samarahan, Malaysia \\ jannisa@unimas.my \\ Intan Zaurah Mat Darus \\ School of Mechanical Engineering \\ Universiti Teknologi Malaysia \\ Johor, Malaysia \\ intan@utm.my
}

\begin{abstract}
Flexible plate structure is a chosen technology used for many applications since past decades ago. However, this structure has a disadvantage that needs to be avoided which is easy to vibrate. Thus, this project presents the modelling of horizontal flexible plate system using bio-inspired flower pollination algorithm. The objective is to obtain an accurate model of the real system in the simulated environment. The collected of real vibration data through experimental study was then utilized to develop the dynamic system model based on linear autoregressive with exogenous (ARX) model structure and optimized by flower pollination algorithm (FPA). The algorithm is a novel bio-inspired optimization algorithm that mimics the real-life processes of the flower pollination. The gained model in this simulation is approved utilizing the most minimal mean squared error, correlation tests, and pole zero graph stability due to check the robustness of the model. The performance of the developed model was then compared with the conventional algorithm known as recursive least square (RLS). The best model achieved in this study will be used as a platform of controller development using active vibration control technique.
\end{abstract}

Keywords-recursive least square, flower pollination algorithm, evolutionary swarm algorithm, system identification, flexible plate structure

\section{INTRODUCTION}

In the past several decades, a flexible structure is widely used in various applications. Based on previous researches, this structure has been used in various study such as fluid, chemical, spacecraft and many more [1-4]. Different elements will be implemented to the structure like frames, shells, beams, and plates for different applications in order to produce good effect results [5]. Flexible structure systems are known to demonstrate an intrinsic property of vibration when subjected to disturbance forces, leading to components and structural damage [6].

The thin rectangular plate structures are the most commonly used in the industrial applications such as bridge decks, solar panels, airport baggage transport conveyor and electronic circuit board design [5]. Most of the industry's applications use flexible structure compared to rigid structures due to its advantages such as lightweight, reliable and high efficiency. In some reasons, the advantages can be the downside of a flexible structure. The lightweight of the flexible structure can be easily influenced by the vibration which will cause damage to the structure [7]. Thus, the unwanted vibration should be removed to sustain an effective system.

In order to obtain a better controller, a proper model of the system need to be modelled at initial. Recently, one of the well-known methods has been given attention by the researchers, which is using system identification technique. System identification is a candidate used to describe mathematical tools and algorithms that build dynamical models from measured data. The practical application domains include pattern recognition, time-series prediction, Boolean function generation, and symbolic regression [6].

System identification had experienced an evolution from time to time. Previously, the methods used to model a structure are Finite Difference and Finite Element methods which are also categorized in system identification. In some time, the conventional algorithm is gradually prefaced as one of the methods to represent the modelling. The latest system identification that had been developed by the researchers is an evolutionary swarm algorithm which is bio-inspired the reallife processes. For instances, the algorithms that recently done by the researchers are particle swarm optimization (PSO), artificial bee colony (ABC), bat algorithm (BAT), flower pollination, firefly and many more.

Recently, a great attention has been received by flower pollination algorithm due to its ability in solving computationally complex and mathematically intractable problems. Inspired by the thoughts of previous research, thus, this study presents the modeling of a horizontal flexible plate structure using evolutionary swarm algorithm via flower pollination algorithm (FPA) and the performance of proposed method will be compared with the conventional algorithm known as recursive least square (RLS).

\section{EXPERIMENTAL SETUP}

An information securing framework is utilized as an accumulation of programming and equipment to gauge or control the physical qualities of a framework. A total information procurement framework comprises of DAQ equipment, sensors and actuators, signal conditioning equipment, and a PC running DAQ programming. Along these lines, from the test arrangement, the info yield vibration information were gathered for flat adaptable plate structure framework. The exploratory apparatus included the parts of mechanical, electrical, and hardware which are work 